\title{
Ovariectomía en Perras: Comparación entre el Abordaje Medial o Lateral
}

\author{
Ovariectomy in Dogs: Comparison between Medial and Lateral Approach \\ Juan L. Masacheㄹ, Mónica C. Brito ${ }^{1}$, Christian F. Sagbay ${ }^{1}$, Pedro G. Webster ${ }^{1}$, \\ F. Patricio Garnica ${ }^{1}$, Carlos Mínguez ${ }^{1,2}$
}

\section{Resumen}

Se evaluó la técnica de ovariectomía mediante abordaje medial o lateral como método para la esterilización en perras. Las variables de medición fueron el tiempo total empleado en la cirugía, la cuantificación de hemorragia durante la intervención y el grado de dolor evaluado a las 2, 4, 6, 18 y 24 horas poscirugía siguiendo el baremo de la escala reducida de Glasgow. Se trabajó con 70 hembras aparentemente sanas, distribuidas de forma aleatoria en dos grupos de 35. El grupo 1 fue esterilizado mediante ovariectomía con abordaje lateral (flancotomía) y el grupo 2 con abordaje medial (celiotomía). En el análisis estadístico se utilizó un modelo de ajuste aditivo, considerando los efectos: forma de abordaje, y peso, edad, raza y número de partos del animal. Las estimas de las diferencias entre técnicas quirúrgicas se obtuvieron mediante mínimos cuadrados generalizados. El registro de datos quirúrgicos y de las observaciones posteriores fue tomado por la misma persona. Los resultados indicaron diferencias significativas para técnica de abordaje y peso y edad del animal. Para la variable tiempo, la flacotomía fue significativamente más rápida. No se observaron diferencias significativas para las variables cuantificación de la hemorragia o grado de dolor entre las dos técnicas. Los resultados demuestran que la ovariectomía mediante flancotomía es un método que requiere de menos tiempo quirúrgico.

Palabras clave: ovariectomía, celiotomía, flancotomía, esterilización, tiempo quirúrgico, grado de dolor

\section{Abstract}

The aim of this study was to evaluate the ovariectomy technique by lateral or medial approach as a sterilization method in bitches. The variables were surgical time, quantification of bleeding during surgery and degree of pain assessed at 2, 4, 6, 18 and

\footnotetext{
${ }^{1}$ Carrera de Medicina Veterinaria y Zootecnia, Universidad Politécnica Salesiana, Cuenca, Ecuador

${ }^{2}$ E-mail:cminguez@ups.edu.ec
}

Recibido: 13 de noviembre de 2015

Aceptado para publicación: 23 de marzo de 2016 


\begin{abstract}
24 hours after surgery, following the short form of the Glasgow composite pain scale. A total of 70 apparently healthy females were selected and randomly distributed in two groups. Group 1 was sterilized by lateral approach ovariectomy (paracostal) and Group 2 by medial approach ovariectomy (celiotomy). In the statistical analysis, the additive effects in the model were: approach technique and weight, age, breed and parity of the animal. Estimates of the differences between surgical methods were obtained by generalized least square. Data recording of the surgical procedure and observations after surgery were carried out by the same person. The results showed statistical differences for the approach technique and animal weight and age. For the variable surgical time, the lateral approach ovariectomy was significantly quicker. None statistical differences between techniques were found due to quantification of bleeding and degree of pain. These results indicated that ovariectomy by lateral approach is a procedure that requires less surgical time.
\end{abstract}

Key words: ovariectomy, celitomy, paracostal, sterilization, surgical time, degree of pain

\section{INTRODUCCIÓN}

La esterilización quirúrgica de perros y gatos es una de las intervenciones más comunes en la práctica veterinaria, siendo además, el principal método anticonceptivo para evitar el crecimiento desmedido de la población de mascotas (Howe, 2006). Una de las técnicas quirúrgicas más empleadas para realizar la esterilización es la ovariectomía (OVE) o extracción de ovarios, que debe ser realizada por un médico veterinario bajo anestesia (van Goethem et al., 2006).

Existen estudios que muestran la preferencia de la OVE sobre la ovariohisterectomía (extirpación de ovarios y útero, $\mathrm{OVH}$ ), dado que solo se requieren incisiones más pequeñas y un menor tiempo de operación y de anestesia (Okkens et al., 1997). Si bien la técnica tradicional para castrar a las perras es la OVH (Stone, 2006; van Goethem et al., 2006; Silva et al., 2007), no hay ventajas definitivas para remover el útero más los ovarios durante la castración (Stone, 2006). Asimismo, se ha demostrado que la OVE, en animales saludables y sin previo tratamiento hormonal, no presenta riesgos notorios en el tracto reproductivo (Okkens et al., 1997; Gadelha et al., 2004; Howe, 2006); sin embargo, es una técnica poco utilizada en el Ecuador.
Existen dos formas de abordar la OVE: la medial (o celiotomía) y la lateral (o flancotomía). La práctica de uno u otro método reside más en la preferencia del operador que en otros aspectos clínicos, pese a que se ha comprobado que el tiempo que dura la operación afecta significativamente al ratio de morbilidad, dolor del animal y a las complicaciones posoperatorias (Case et al., 2011).

El objetivo de este trabajo fue evaluar la OVE medial y lateral como métodos más apropiados para la esterilización de hembras caninas, mediante la comparación de tiempo total empleado, cualificación de hemorragia durante el acto quirúrgico y grado de dolor con el fin de definir el tipo de abordaje más apropiado en este tipo de esterilizaciones.

\section{Materiales y Métodos}

\section{Animales}

El trabajo se realizó siguiendo los parámetros de asepsia, antisepsia, desinfección, esterilización e higienización establecidos en la sección de clínica quirúrgica de la Clínica Veterinaria «PoliVet» de la Universidad Politécnica Salesiana, en la ciudad de Cuenca, Ecuador. 
La clínica cuenta con todos los permisos expedidos por Agrocalidad (institución pública adscrita al Ministerio de Agricultura, Ganadería, Acuacultura y Pesca, encargada de la definición y ejecución de políticas de control y regulación para la protección y el mejoramiento de la sanidad animal) necesarios para realizar la práctica de Medicina Veterinaria.

El estudio involucró 70 perras aparentemente sanas, sin distinción de raza, tamaño, peso, número de partos y edad. La anamnesis indicó ausencia de problemas reproductivos, administración de anticonceptivos, y la evaluación clínica mostraba un aparato reproductor sano al momento de la cirugía. El periodo experimental se desarrolló desde diciembre de 2014 hasta julio de 2015.

\section{Diseño Experimental}

El protocolo de pre-anestesia estuvo compuesto por sulfato de atropina $(0.04 \mathrm{mg} /$ $\mathrm{kg})+$ maleato de acepromacina $(0.05 \mathrm{mg} /$ $\mathrm{kg})+$ xilacina $(1.1 \mathrm{mg} / \mathrm{kg})$ en dosis bolo vía intramuscular; y el de anestesia regional (epidural) por lidocaína sin epinefrina $(20 \mathrm{mg} /$ $3.5 \mathrm{~kg}$ ), vía epidural. Los animales fueron distribuidos de forma aleatoria en los dos grupos hasta completar 35 hembras por grupo.

\section{Grupo 1 (OVE con abordaje lateral [flancotomía]):}

Las fases quirúrgicas para realizar la esterilización fueron:

- Fase 1. Abordaje de la pared abdominal. Con el animal en posición decúbito lateral derecho, se realizó una incisión en la piel al lado derecho de $1 \mathrm{~cm}$ a $5 \mathrm{~cm}$ caudal a la última costilla y por debajo de las apófisis transversas lumbares, con una longitud de $1 \mathrm{~cm}$ a $4 \mathrm{~cm}$ siguiendo una dirección longitudinal. El tejido subcutáneo se incidió mediante disección roma con tijera Metzembaun, al igual que en los planos musculares (oblicuo abdo- minal externo, oblicuo abdominal interno y transverso abdominal) y el peritoneo. Los músculos fueron separados de acuerdo a la dirección de sus fibras, mediante separación digital, al igual que el peritoneo.

- Fase 2. Ubicación y extracción de los ovarios. En primer lugar se ubicó el ovario izquierdo, se rompió el ligamento suspensorio del ovario, se tomó el pedículo ovárico con una pinza hemostática; se colocó otra pinza hemostática en la porción de cuerno uterino inmediatamente adyacente al ovario, se procedió a extirpar el ovario ubicado entre las dos pinzas; se realizó angiotripsia del pedículo ovárico (promedio entre 10 y 12 vueltas). Posteriormente, se suturó mediante un patrón de transfixión la porción de cuerno uterino libre, utilizando material de sutura polifilamento absorbible de poliglactina 910 (Vicryl Plus Antibacteria, Johnson \& Johnson) de un calibre adecuado.

Se procedió en forma similar con el ovario derecho. Se le ubicó guiándose por el cuerpo y cuerno uterino correspondiente.

- Fase 3. Síntesis de la laparotomía. Luego de la extirpación de los ovarios, se suturaron las capas musculares y tejido subcutáneo en un solo plano, y la piel por separado. El material de sutura utilizado para los planos muscular y subcutáneo fue el mismo que en que en la fase 2. El patrón de sutura empleado fue continua anclada, para ambos planos.

\section{Grupo 2 (OVE con abordaje medial [celiotomía]):}

- Fase 1. Abordaje de la cavidad abdominal. Con el animal en posición ventrodorsal, y tras vaciar la vejiga de forma manual, se realizó una incisión en la piel desde el ombligo hasta aproximadamente la mitad del último par mamario o hueso púbico, dependiendo del tamaño 
del animal. En perras obesas fue necesaria en ciertas ocasiones alargar la incisión cranealmente para permitir la suficiente exposición del pedículo ovárico.

- Las fases 2, 3 y 4 explicadas para el Grupo 1 fueron similares en este grupo.

Además, se utilizó una única aplicación postquirúrgica de un analgésico antiinflamatorio no esteroidal, ketoprofeno $1 \mathrm{mg} / \mathrm{kg}$, vía intramuscular, penicilina + estreptomicina (15.000 UI/kg peso vivo) y aplicación tópica de Yodo Povidona 10\% una vez al día.

\section{Datos y Análisis Estadístico}

Se registró el tiempo empleado en la cirugía (en minutos), se cuantificó la hemorragia durante el acto quirúrgico (leve, moderada o abundante), de acuerdo a la cantidad de gasas y compresas utilizadas y a la observación visual durante el transcurso del acto quirúrgico (Silva et al., 2007), y el grado de dolor a las 2, 4, 6, 18 y 24 h después de la cirugía según la escala reducida de Glasgow (The Scale-Form Glasgow Composite Measure Scale [CMPS-SF]), según Reid et al. (2007). Esta escala valora el grado de dolor en un rango de 0 a 20.

La estimación de las diferencias entre los dos métodos de esterilización se realizó con el siguiente modelo, $\mathrm{Y}_{\mathrm{ijk} \mathrm{km}}=\mathrm{T}_{\mathrm{i}}+\mathrm{R}_{\mathrm{j}}+\mathrm{P}_{\mathrm{k}}$ $+\mathrm{E}_{1}+\mathrm{N}_{\mathrm{m}}+\mathrm{e}_{\mathrm{ijklm}}$, donde: $\mathrm{Y}_{\mathrm{ijklm}}$ es el dato evaluado; $\mathrm{T}_{\mathrm{i}}$ es la variable técnica quirúrgica utilizada (flancotomía o celiotomía); $R_{j}$ es la variable raza, divida en once categorías; $\mathrm{P}_{\mathrm{k}}$ es la variable peso ( 0 a $5,5.1-10,10.1-15$, $>15.1 \mathrm{~kg}$ ); $\mathrm{E}_{1}$ es la variable edad (0 a 6 meses correspondiente a cachorros, 6.1 a 12 meses correspondiente a perras jóvenes, 12.1 a 72 meses correspondiente a animales adultos, $>72$ meses correspondiente a animales de geriátrico); $\mathrm{N}_{\mathrm{m}}$ es la variable número de partos, y $\mathrm{e}_{\mathrm{ijklm}}$ es el error del modelo. Las categorías de edad del animal corresponderían a cachorros, jóvenes, adultos y geriátricos.
Las diferencias entre técnicas quirúrgicas se determinaron mediante mínimos cuadrados generalizados utilizando el programa estadístico R Project (R Core Team, 2013), calculando los valores medios mínimos cuadráticos con el paquete lsmeans. Valores de $\mathrm{P}$ inferiores a 0.05 se consideraron significativos.

Para evitar el posible sesgo en los datos debido al efecto operador, todos los procedimientos quirúrgicos, así como la información de evaluación cualitativa, fue realizada por el mismo médico veterinario, con gran experiencia en las intervenciones de esterilización mediante los dos procedimientos evaluados. Asimismo, se aplicó la misma dosis de anestésico por peso vivo $(20 \mathrm{mg} / 3.5 \mathrm{~kg}$ peso vivo), vía epidural, en todos los casos, para evitar sesgos en las muestras de dolor debida a la dosis aplicada.

\section{Resultados y Discusión}

Para la variable tiempo total, los factores que presentaron significancia estadística fueron la técnica, el peso y la edad, no resultando efectos significativos las variables raza o número de partos.

Las medias mínimo cuadráticas muestran que el tiempo empleado en realizar una OVE con abordaje lateral fue significativamente más rápido que el abordaje ventral (Cuadro 1). A su vez, el tiempo necesario para realizar la esterilización se incrementó con el peso del animal (Cuadro 1) aunque estas diferencias no fueron significativas entre los grupos intermedios. Asimismo, la OVE requirió mayor tiempo de ejecución en el grupo de menor edad (0-6 meses) en comparación con las hembras de mayor edad (Cuadro 1).

Asimismo, no se observaron diferencias significativas entre las técnicas quirúrgicas en relación al grado de hemorragia. Solo se presentaron dos casos de hemorragias mo- 
Cuadro 1. Media mínimo cuadrática de la variable Tiempo Quirúrgico (en minutos), según peso y edad de los animales y técnicas de abordaje empleadas en la práctica de la ovariectomía en perras

\begin{tabular}{llcccc}
\hline Variable & & $\mathrm{N}^{1}$ & LSMean $^{2}$ & $\mathrm{DE}^{3}$ & $\mathrm{IC}^{4}$ \\
\hline $\begin{array}{l}\text { Técnica } \\
\text { quirúrgica }\end{array}$ & $\begin{array}{l}\text { Lateral } \\
\text { (flancotomía) }\end{array}$ & 35 & $23.48^{\mathrm{a}}$ & 0.97 & $21.53-25.43$ \\
& $\begin{array}{l}\text { Ventral } \\
\text { (celiotomía) }\end{array}$ & 35 & $28.85^{\mathrm{b}}$ & 1.08 & $26.68-31.02$ \\
& Peso (kg) & 15 & $21.97^{\mathrm{a}}$ & 1.19 & $19.64-24.29$ \\
& 0 5 & 17 & $26.78^{\mathrm{b}}$ & 1.08 & $24.61-28.96$ \\
& 5.1 a 10 & 17 & $27.59^{\mathrm{b}}$ & 1.17 & $25.22-29.96$ \\
& 10.1 a 15 & 16 & $32.31^{\mathrm{c}}$ & 1.05 & $30.26-34.35$ \\
Edad (meses) & 0 a 6 & 15 & $33.18^{\mathrm{a}}$ & 1.49 & $30.28-36.08$ \\
& 6 a 12 & 17 & $27.08^{\mathrm{b}}$ & 1.33 & $24.49-29.67$ \\
& 12 a 72 & 19 & $26.66^{\mathrm{b}}$ & 1.20 & $24.32-29.00$ \\
& $>72$ & 14 & $26.05^{\mathrm{b}}$ & 1.52 & $23.09-29.01$ \\
\hline
\end{tabular}

${ }^{1} \mathrm{~N}$ : número de animales; ${ }^{2}$ LSMean: valor medio mínimo cuadrático de la variable tiempo (minutos); ${ }^{3} \mathrm{DE}$ : desviación estándar; ${ }^{4} \mathrm{IC}$ : intervalo de confianza.

a,b Superíndices diferentes dentro de columna y en cada variable indican diferencia significativas $(p \varangle 0.05)$

deradas en la flancotomía y un caso en la celiotomía, habiendo hemorragias leves en el resto de intervenciones quirúrgicas. Por otro lado, tampoco se encontraron diferencias significativas para el grado de dolor, habiéndose registrado valores de 2.1 y 2.3 (escala de 0 a 20) en promedio a las 2 horas de la cirugía por flancotomía y celiotomía, respectivamente y disminuyendo gradualmente con el transcurso de las horas.

En la literatura no existe uniformidad de criterios sobre el mejor método de abordaje para realizar una OVE (Silva et al., 2007). Está demostrado en varios estudios en perros que el incremento de la duración de una intervención quirúrgica en general, y una esterilización en particular, aumenta la probabilidad de complicaciones, estrés y daño posoperatorio de los pacientes (Devitt et al., 2005; Case et al., 2011).

En el presente estudio, la flancotomía fue más rápida y esto podría ser debido a diversas causas en el proceso quirúrgico (Verstegen, 2000). La primera es la menor necesidad de cortes para llegar a la cavidad abdominal, ya que luego de cortar la piel, solo se realiza una incisión roma mediante pinza en la flancotomía, mientras que en la celiotomía se requiere cortar la línea alba para llegar a la cavidad abdominal. Además, en la flancotomía se requiere de menos puntos de sutura pues el corte es de menor tamaño, en tanto que en la celiotomia se debe tener mayor cuidado pues las vísceras abdominales ejercen presión sobre la sutura y en el caso de mala praxis se podrían producir hernias en el paciente. 
A nivel de peso, los resultados coinciden con Silva et al. (2007), quienes obtuvieron un incremento en el tiempo total de la cirugía a medida que aumentaba el peso de los animales. Este efecto es comprensible porque a mayor peso se demanda mayor tiempo en cada una de las fases operatorias, así como más complicaciones para manipular el animal en la camilla.

El mayor tiempo que demandó la cirugía en las perras cachorras, indistintamente de la técnica de abordaje, se debió al mayor cuidado que demandan, pues su tracto reproductivo es pequeño y poco desarrollado. Estos resultados están en concordancia con Vertegen (2000) y Silva et al. (2007).

Una variable de importancia para decidir el mejor tipo de abordaje es el grado de dolor que puede ocasionarse con la intervención quirúrgica. Actualmente, existen normativas bien desarrolladas acerca del bienestar animal (RSPCA, 2007) y es deber de todo facultativo veterinario utilizar la técnica quirúrgica que menor grado de dolor proporcione a los pacientes. En la valoración del grado de dolor se utilizó la escala reducida de Glasgow, el CMPS-SF (Reid et al., 2007), que está basada en observar el comportamiento de los perros para evaluar el dolor agudo (Holton et al., 2001) y en el uso de un cuestionario que consta de siete categorías de comportamiento (la postura, la actividad, la vocalización, atención a la herida o zona dolorida, comportamiento, la movilidad, y la respuesta al toque). Es importante hacer notar que el CMPS-SF es la única escala de dolor que ha sido validada para la evaluación del dolor agudo en perros (Holton et al., 2001; Morton et al., 2005; Murrell et al., 2008).

Los resultados no mostraron diferencias significativas con respecto al grado de dolor según la técnica quirúrgica empleada. Esto puede deberse a que las muestras de dolor hasta las 24 horas de la intervención quirúrgica están determinadas por el tipo y dosis de anestésico utilizado y por el tipo de tratamiento recetado para el proceso posquirúrgico (Jin y Chung, 2001; Hansen, 2005; Valtolina et $a l ., 2009)$. En el presente estudio, todos los animales fueron anestesiados con el mismo compuesto activo, se les aplicó la misma dosis y siguieron el mismo tratamiento posquirúrgico, independientemente de cómo se realizó el abordaje a la cavidad abdominal, de allí que no hubiera diferencias en términos de dolor.

\section{Conclusiones}

Los resultados indican que el abordaje lateral (flancotomía) en la técnica de ovariectomía en perras requiere un menor tiempo quirúrgico frente al abordaje ventral (celitomía).

\section{Literatura Citada}

1. Case JB, Marvel SJ, Boscan P, Monnet EL. 2011. Surgical time and severity of postoperative pain in dogs undergoing laparoscopic ovariectomy with one, two, or three instrument cannulas. J Am Vet Med Assoc 239: 203208. doi: 10.2460/javma.239.2.203

2. Devitt CM, Cox RE, Hailey JJ. 2005. Duration, complications, stress, and pain of open ovariohysterectomy versus a simple method of laparoscopic-assisted ovariohysterectomy in dogs. J Am Vet Med Assoc 227: 921-927.

3. Gadelha C, Ribeiro A, Apparício M, Covizzi GC, Vicente WRR. 2004. Acquired vesicovaginal fistula secondary to ovariohysterectomy in a bitch: a case report. Arq Bras Med Vet Zootec 56: 183-186. doi: 10.1590/S010209352004000200007

4. Hansen BD. 2005. Analgesia and sedation in the critical ill. J Vet Emerg Crit Care 15: 285-294. doi: 10.1111/ j.1476-4431.2005.00166.x

5. Holton L, Reid J, Scott EM, Pawson P, Nolan A. 2001. Development of a behaviour-based scale to measure acute 
pain in dogs. Vet Rec 148: 525-531. doi: 10.1136/vr.148.17.525

6. Howe LM. 2006. Surgical methods of contraception and sterilization. Theriogenology 66: 500-509. doi: 10.1016/j.theriogenology.2006.04.005

7. Jin F, Chung F. 2001. Multimodal analgesia for postoperative pain control. J Clin Anesth 13: 524-539. doi: 10.1016/S09528180(01)00320-8

8. Morton CM, Reid J, Scott EM, Holton LL, Nolan AM. 2005. Application of a scaling model to establish and validate an interval level pain scale for assessment of acute pain in dogs. Am J Vet Res 66: 2154-2166. doi: 10.2460/ ajvr.2005.66.2154

9. Murrell JC, Psatha EP, Scott EM, Reid J, Hellebrekers LJ. 2008. Application of a modified form of the Glasgow pain scale in a veterinary teaching centre in the Netherlands. Vet Rec 162: 403-408. doi: 10.1136/ vr.162.13.403

10. Okkens AC, Kooistra HS, Nickel RF. 1997. Comparison of long-term effects of ovariectomy versus ovariohysterectomy in bitches. J Reprod Fertil 51: 227231.

11. R Core Team. 2013. R: A language and environment for statistical computing. Vienna, Austria: R Foundation for Statistical Computing. [Internet]. Disponible en: http://www.R-project.org/

12. Reid J, Nolan AM, Hughes JML, Lascelles D, Pawson P, Scott EM. 2007. Development of the short-form Glasgow Composite Measure Pain Scale
(CMPS-SF) and derivation of an analgesic intervention score. Anim Welfare 16: 97-104.

13. RSPCA [Royal Society for the Prevention of Cruelty to Animals]. 2007. Measuring Animal Welfare in the UK. Horsham: RSPCAVerstege. [Internet]. Disponible en: http:// www.rspca.org.uk/home

14. Silva R, Grajales N, Mejía R, Loaiza A. 2007. Evaluación de ovariectomía mediante abordaje paracostal y angiotripsia, como método de esterilización en caninos. Vet Zootec 1: 29-35.

15. Stone EA. 2006. Ovario y útero. En: Slatter D (ed). Tratado de cirugía de pequeños animales. $3^{\mathrm{a}}$ ed. Buenos $\mathrm{Ai}$ res: Inter-Médica. p 1709-1729.

16. Valtolina C, Robben JH, Uilenreef J, Murrell JC, Aspegrén J, McKusick BC, Hellebrekers LJ. 2009. Clinical evaluation of the efficacy and safety of a constant rate infusion of dexmedetomidine for postoperative pain management in dogs. Vet Anaesth Analg 36: 369-383. doi: 10.1111/j.1467-2995.2009.00461.x

17. van Goethem B, Schaefers-Okkens A, Kierpenstijn J. 2006. Making a rational choice between ovariectomy and ovariohysterectomy in the dog: a discussion of the benefits of either technique. Vet Surg 35: 136-143.

18. Verstegen J. 2000. Contraception and pregnancy termination. In: Ettinger S, Feldman E (eds). Textbook of veterinary internal medicine diseases of the dog and cat. $5^{\text {th }}$ ed. California, USA: WB Saunders Company. p 1542-1543. 